\title{
Equilibrios inestables para el periodismo para la paz en conflictos nacionalistas
}

Equilíbrios instáveis para o jornalismo para a paz em conflitos nacionalistas

Unstable balances for peace journalism in nationalist conflicts

Xavier Giró

- Universitat Autónoma de Barcelona

- Profesor titular de Periodismo Político en Universitat Autónoma de Barcelona

- Director del grupo de investigación Observatori de la Cobertura de Conflictes (OCC)

- Codirector de la diplomatura de postgrado La Comunicación de los Conflictos y la Paz

- Doctor en Periodismo y master in Journalism and Public Affairs por la American University (Washington DC, Estados Unidos)

- Lineas de investigación: medios e inmigración, y medios y conflictos armados

- Especialista en Peace Journalism y en el análisis de la cobertura informativa de conflictos

- e-mail:xavier.giro@uab.cat 


\section{Resumen}

El periodismo de paz tiene un reto particular en la cobertura informativa de los conflictos nacionalistas, a saber, como contribuir a una solución justa - y por lo tanto a favor de los oprimidos - y a la vez evitar que ese compromiso contribuya a una escalada de la confrontación hacia la violencia, algo extremadamente fácil en disputas tan cargadas de sentimientos y arraigadas en las culturas de los pueblos. Los investigadores en periodismo de paz hacen frente a un reto paralelo: estudiar si el discurso sobre el conflicto contribuye a resolverlo de la forma más justa posible y con el menor sufrimiento.

\section{PALABRAS CLAVE: PERIODISMO DE PAZ•NACIONALISMO • CONFLICTOS•DISCURSO.}

\section{Abstract}

The peace journalism has a particular challenge in the informational coverage of nationalist conflicts, namely, how to contribute to a fair solution and, therefore, in favor of the oppressed, and, at the same time, to prevent that this commitment contributes to an escalation of the confrontation towards violence, something extremely easy in disputes so full of feelings and rooted in the culture of peoples. Researchers in peace journalism face a parallel challenge: to study whether the discourse on the conflict contributes to transform it in the fairest way possible and with the least pain.

KEYWORDS: PEACE JOURNALISM • NATIONALISM • CONFLITOS • DISCOURSE.

\section{Resumo}

O jornalismo para a paz tem um desafio particular na cobertura informativa dos conflitos nacionalistas, a saber, como contribuir para uma solução justa e, portanto, a favor dos oprimidos e, ao mesmo tempo, evitar que esse compromisso contribua para uma escalada da confrontação em direção à violência, algo extremamente fácil em disputas tão carregadas de sentimentos e arraigadas nas culturas dos povos. Os pesquisadores em jornalismo para a paz enfrentam um desafio paralelo: estudar se o discurso sobre o conflito contribui para transformá-lo da forma mais justa possível e com o menor sofrimento. 


\section{INTRODUCCIÓN}

$\mathrm{E}$ n el terreno de los conflictos nacionalistas, los medios de comunicación son capaces de levantar o de hundir casi cualquier reivindicación. Pueden difuminar los agravios recibidos o crear la percepción de que hay agravios que, en realidad, son inexistentes. También tienen a su alcance tanto fortalecer como debilitar la conciencia colectiva de una comunidad. Y, de igual modo, está en sus manos incitar al odio y la violencia entre los grupos enfrentados o bien actuar en la dirección opuesta.

Cubrir un conflicto comporta no solo exponer las disyuntivas que plantea su desarrollo, sino buscar respuestas y trabajar en coherencia con ellas. Implica, de hecho, tomar una posición sobre si las demandas de una comunidad son, o no, justas 0 en qué medida lo son; sobre si se está a favor de que en un grupo humano haya algún tipo de conciencia colectiva, incluida la nacional, y sobre si la dinámica con que se debe gestionar el conflicto debe ser, o no, violenta.

Y siempre hay una posición, aunque no sea explícita. Por poner un ejemplo, ¿acaso no es una posición la que se autodenomina 'neutral' en un contexto de injusticia?

El reto para el periodismo de paz está en desplegar una cobertura comprometida contra el abuso de poder y, en general, contra la injusticia, y llevarla a cabo de tal forma que fomente la resolución pacífica del conflicto, que evite su escalada hacia la violencia.

Correlativamente, para el o la investigadora-analista de la cobertura de conflictos que tome una perspectiva crítica, su objetivo no solo está en estudiar técnicamente cómo ha sido el trabajo periodístico, sino que, fundamentalmente, mira, en primer lugar, si el discurso desplegado por el medio de comunicación deslegitima el abuso de poder - es decir, si apoya una reivindicación justa contra un agravio - y, en esa medida, favorece a la justicia y, en segundo lugar, si construye la paz. Sobre estos puntos girarán sus hipótesis.

\section{LEGITIMIDAD Y RETOS}

En todos los conflictos, pero especialmente en los nacionalistas, la valoración de una reivindicación o de un agravio puede ser una cuestión bastante abierta, aunque no siempre. Por ejemplo, las masacres cometidas contra minorías ofrecen pocas dudas. No ocurre lo mismo con las políticas lingüísticas o las disputas sobre inversiones o los impuestos que afectan a una parte de la población. También es un asunto abierto a debate si una comunidad es o no una 'nación' y si tiene derecho a la autodeterminación. Consecuentemente, será relevante considerar si conviene o no que arraigue o se expanda no solo la conciencia de nación, sino también qué tipo de conciencia hegemónica nacional es la que cuaja porque, por poner un caso, no es lo mismo un nacionalismo xenófobo que un nacionalismo intercultural.

Tanto la cuestión de los agravios como la de la conciencia nacional comportan una postura ideológica y, en gran medida, están abiertas a la polémica. El problema relativo a la gestión del conflicto, aunque también es ideológica, es menos abierta, más circunscrito.

Si bien, tanto la defensa de una reivindicación como su negación, o el fomento de una conciencia colectiva o de otra, pueden ser - no quiere decir que lo sean siempre - legítimas, bajo la perspectiva de avanzar hacia la resolución justa del conflicto. No es legítimo ni conveniente, por ejemplo, que se fomente una representación distorsionada de la confrontación, ni de los actores, ni de sus acciones, ni de los problemas en disputa. Tampoco lo es el uso del discurso para justificar el odio o la 
violencia contra de los otros. Es decir, puede ser legítimo defender ideas, proyectos, reivindicaciones, identidades, pero no de cualquier manera. El dilema en este terreno está más acotado.

Así pues, si estudiamos los discursos de los actores - incluidos los medios, ya que en definitiva también son actores - y consideramos que la persecución de sus objetivos no justifica la distorsión discursiva ni el fomento del odio contra el oponente ni el recurso a la violencia porque ambos causan un gran sufrimiento (superior al existente) y todos, juntos y por separado, alejan o impiden acuerdos y soluciones justas, es lógico que señalemos qué elementos del periodismo favorecen o bien frenan la escalada hacia estadios indeseables.

Los dilemas y el reto de superarlos son continuos. Luchar es aumentar la tensión. No moverse es claudicar. ¿Cómo contribuir a la máxima democracia y justicia y, a la vez, al mínimo sufrimiento? ¿Cómo perseguir la máxima satisfacción de las reivindicaciones y la mínima imposición?

La práctica periodística comprometida se encuentra en un equilibrio de frágil estabilidad, sometida a presiones de todos los actores, con el deber de valorar todas las reivindicaciones, las propuestas y, sobre la marcha del conflicto, intentando no contribuir a la escalada hacia la violencia.

Este artículo se propone exponer prácticas discursivas periodísticas que intervienen en el desarrollo de confrontaciones nacionalistas e ilustrarlas con ejemplos de diferentes conflictos sin hablar ni de Cataluña ni de España, lugar donde el autor reside. En primer lugar, se abordarán las descripciones de los actores que los medios elaboran o difunden; en segundo lugar, de los problemas, y, en tercer lugar, del proceso de negociación o de gestión del conflicto.

Las concepciones recíprocas que tienen los contrincantes son factores determinantes de las expectativas con que se aborda la confrontación. El desconocimiento entre los actores o los grupos sociales involucrados, a menudo debido al fomento de los estereotipos, y la identificación errónea o ambigua de los sujetos-actores y de sus responsabilidades son serios obstáculos para encontrar salidas.

En Bélgica, por ejemplo, el sistema comunicativo está claramente diferenciado. Hay medios en francés para los valones y en neerlandés para los flamencos. No hay medios -ni diarios, ni radios, ni televisiones- dirigidos a ambas comunidades. Cada una tiene su propia organización audiovisual regulada por la comunidad lingüística y no por el gobierno central. Ambas promueven la identidad cultural de su propia parte.

Las radios comerciales son igualmente exclusivas. Prácticamente en ningún medio hay información sobre la otra comunidad. Sólo un 3\% de las noticias de la televisión flamenca tienen que ver con asuntos de la valona. Es cierto que los canales franceses ponen un poco más de atención a Flandes y al estado central, pero de los pocos políticos francófonos que aparecen en la televisión flamenca, el $90 \%$ tienen cargos federales.

La contrapartida está en la simplificación y los estereotipos que fomentan los medios de ambos lados. El foco informativo se coloca sobre las diferencias, como si fuesen caricaturas. Cada comunidad se percibe como víctima de la otra. Flandes aparece repetidamente como un nido de fascistas, de gente de extrema derecha, que pretenden independizarse de los valones. Los mecanismos son habituales: las declaraciones escandalosas de un político separatista se adjudican a menudo a todos los políticos y de aquí a toda la comunidad. Las declaraciones que subrayan las diferencias son amplificadas.

En sentido inverso, los valones son representados como vagos y parásitos socialistas, cargados de escándalos y corrupción. Las zonas ricas y de voto liberal de Valonia son ignoradas por los medios flamencos. También, como en un juego de espejos 
con respecto a la otra comunidad, las declaraciones flamígeras de algún líder francófono son proyectadas como si fuesen del conjunto de su comunidad.

Algo similar ocurre en Canadá. Las visiones del país aportadas por los medios varían sustancialmente ya sean en inglés 0 en francés. Como también cambian si los medios son del centro (o centros) o de las regiones. Igualmente son distintas las visiones que uno tiene si es miembro de las Primeras Naciones, o descendiente de colonos, o un refugiado reciente de Liberia 0 del Vietnam.

A pesar de las dos visiones diferenciadas que los medios ingleses o franceses aportan sobre las nociones de nacionalidad, identidad nacional y nacionalismo, a raíz del referéndum de soberanía en Québec en octubre de 1995 y de las quejas del primer ministro canadiense del momento, un estudio independiente del servicio francés de la Canadian Broadcasting Corporation/Radio Canadá concluyó que, aunque cada una de la redes de televisión usara filtros diferentes, no se podía decir que estuviesen políticamente sesgadas. De todas maneras, dos meses más tarde, los programas satíricos tradicionales de fin de año de las dos cadenas estuvieron decorados con gags de mal gusto, casi racistas con descripciones estereotípicas del otro grupo lingüístico.

La información rigurosa sobre los actores y sobre quién es el responsable de qué no es solo una cualidad del buen periodismo, es un factor que influye en la representación mental que adopta la ciudadanía del conflicto y correspondientemente de los contrincantes, y, por lo tanto, de las batallas que tiene por delante.

En los conflictos nacionalistas, el antagonista puede ser descrito de diversas formas y no todas son exactas ni apropiadas. Por ejemplo, en el caso de la ex-Yugoslavia, un crimen cometido por guerrilleros serbios no es un crimen cometido por 'los serbios', aunque así fue presentado. Lo mismo vale para croatas o bosnianos. Tampoco una agresión protagonizada por soldados del ejército bajo órdenes del entonces presidente Slobodan Milosevic puede ser descrita con una agresión de 'Serbia' o de 'Belgrado'. Por mucho que los agresores actúen con la pretensión de hacerlo en nombre de lo que identifican como su nación -0, salvando las diferencias, en nombre de su creencia religiosa- no se puede hacer responsable a toda la comunidad identificada como nación - o creencia- de aquellas acciones.

En conflictos de alta intensidad de tipo étnico o identitario, las agresiones desde una comunidad (he escrito 'desde' no 'de') son cometidos por una minoría - a veces muy pequeña, a veces simplemente es el gobierno que agrede - contra el conjunto de la otra o contra cualquier miembro de la otra por el simple hecho de ser miembro de la otra comunidad. Así, sí es cierto que toda un comunidad tiene el papel de actor-víctima, pero raramente el de actor-agresora.

En conflictos de menos intensidad, el razonamiento no difiere en esencia. Lo que los líderes hacen/cometen/perpetran 0 realizan no necesariamente lo hace toda la comunidad que ellos lideran o gobiernan. Por lo tanto, las atribuciones colectivas de responsabilidad, tal como se hace a través del nombre plural de los integrantes de la comunidad o nación - con términos como 'los ingleses' o 'los canadienses' o 'los valones' o 'los flamencos' - no ofrecen un buen retrato del conflicto.

Tampoco lo aportan los nombres colectivos singulares que identifican a las comunidades - como, por ejemplo, Inglaterra, Canadá, Valonia, Flandes o los de sus capitales usadas como sinónimo del país para hablar de las declaraciones o acciones del gobierno o de un partido o de un grupo ciudadano. No es excusa alegar que en periodismo hacen falta los sinónimos para no repetir términos que afean el texto, porque aunque, es cierto, hay personas que ya entienden que las denominaciones colectivas han sido usadas solo en un afán estilístico o porque no había más espacio en el titular, en tiempos de confrontaciones, es igualmente cierto que hay personas que no lo perciben y menos aún si algunos líderes se abonan a las generalizaciones contra el enemigo. 
En otro nivel se sitúa la discusión sobre si el responsable de una determinada acción o, dicho de otro modo, el actor preciso que es necesario identificar es el 'Estado' o el grupo político que controla el Estado. No es una cuestión baladí porque para el independentista un argumento de peso a favor de su causa consiste en ver que el problema está en la naturaleza del Estado del que se quiere precisamente independizar. Contrariamente, para el no independentista el problema está en las políticas de quien controla el Estado que oprime, no en ese Estado en sí mismo.

No obstante, también hay independentistas que no arguyen que el problema sea el Estado en sí mismo, sino que en un plazo de tiempo relativamente soportable no es posible desplazar a las fuerzas que controlan el Estado y, por lo tanto, es necesario, o simplemente mejor, dejarlo. Aceptemos que se trata de un aspecto abierto y admitamos, por tanto, que ambas perspectivas pueden plantearse y que no sea rechazable de plano hablar del actor Estado.

Con relación a los problemas alrededor de los que gira el conflicto, los actores tienden a echar mano de la misma estrategia discursiva que usan al hablar de sus oponentes, es decir, la conocida como cuadrado de polarización ideológica: (lado 1) enfatizan los defectos, los errores y las derrotas de los otros y (lado 2) mitigan lo mismo cuando es propio; además, (lado 3) paralelamente enfatizan las cualidades, los aciertos y las victorias propias y (lado 4) mitigan los mismos cuando es del contrario. Si se trata de demandas o de agravios, operan con la misma lógica.

Mitigar o enfatizar se puede lograr sin mentir, simplemente, citando más a unos que a otros. 0 poniendo más el micro frente a la boca de unos que de otros. En definitiva, si los medios dan la voz solo a una parte o sobre todo a una de las partes enfrentadas, está claro que no contribuyen en demasía a la resolución del conflicto. Así fue como operaron algunos grandes medios en Serbia y en Croacia, durante la guerra a principios de los años 90.

Los conflictos nacionalistas también afectan a la Europa de larga tradición democrática. Es el caso, más reciente, de Escocia y su referéndum de autodeterminación. En 2012, en la Gran Bretaña, una información de The Guardian (23 de marzo) titulaba: "David Trimble: Scottish nationalists 'doing violence' to identity of Scots"'. Y de subtítulo: "Former Ulster Unionist leader says all Scots have a dual British identity that is under threat amid push for independence"2 (David..., 2012).

Es tan solo una pieza periodística y su análisis no permite extraer conclusiones sobre el conjunto del discurso del diario, ni es la intención de este comentario. Acotado esto, se puede afirmar sencillamente que al reproducir acríticamente las palabras de un mitin de Trimble, el diario divulga una estrategia discursiva inflamatoria -incendiaria o alarmista, si se prefiere- de una de las partes. Inflamatoria porque el término 'violencia', aunque en el plano académico podría ser aceptable entendido como violencia simbólica, en la esfera pública roza de cerca la semántica de la violencia que la ciudadanía conoce por exceso, la física. Y esa puede ser la compresión para un lector poco académico.

Se podría argumentar que el texto del artículo deshace esta segunda interpretación de 'doing violence'. Sin embargo, para deshacer dudas sobre el punto de vista del artículo, no basta con el lead: "David Trimble has accused Scottish nationalist of "doing violence" to the partly British identity of every Scot by trying to break up the UK"3 (David..., 2012). 'Romper' [break up] es un verbo con carga valorativa negativa en casi todas las culturas.

1 "David Trimble: los nacionalistas escoceses 'ejercen violencia contra la identidad de los escoceses", traducción del autor.

2 "El ex líder unionista del Ulster dice que todos los escoceses tienen una identidad británica dual que está amenazada en la pugna por la independencia", traducción del autor.

3 "David Trimble ha acusado a los nacionalistas escoceses 'de ejercer violencia' contra la identidad parcialmente británica de cada escocés con el intento de romper el Reino Unido", traducción del autor. 
Como solución, no se trata de que los medios silencien a las partes que con sus palabras promueven la escalada. De hecho, si los medios dan la voz a todas las partes enfrentadas, entonces están más cerca de ofrecer una mejor comprensión del conflicto, pero su trabajo se queda a medio camino si no asumen explícita o implícitamente cuales son los problemas y cuáles sus dimensiones. Hay que darlo y hay que discutirlo. En particular, cumplen a medias su tarea si no valoran las acciones y los discursos de los actores en lucha. No basta con transmitir al público el discurso que cada parte despliega en su estrategia política y menos polariza hacia la violencia.

Para finalizar, consideremos cómo se presentan a la audiencia las leyes de un país que afectan a la o a las soberanías (uno de los asuntos más disputados) en conflictos nacionalistas de baja intensidad (en los que no hay guerra). En los conflictos de alta intensidad, no hace falta discutirlo porque manda la ley de las armas. Pues bien, en la lingüística anglosajona se habla de metáforas gramaticales para identificar sustantivos que suplen (o esconden) el resultado de acciones humanas. El discurso de los actores en conflicto usa a menudo este recurso.

Las leyes, sean o no fundamentales dentro de un sistema jurídico, son productos humanos y, por lo tanto, modificables. Todas ellas, tanto las que prescriben la unidad de un país como el código penal. Presentarlas como sujetos de oraciones para decir que prohíben u obligan a hacer lo que sea [por ejemplo, al afirmar que 'La ley establece que el país X es una única nación'], es metaforizar -léase, esconder- que no son las leyes sino los individuos, o los grupos que las aprobaron, quienes prohíben o establecen lo que se puede o no hacer. La metáfora gramatical tiende a naturalizar el resultado de la acción humana con lo que resultaría 'fuera de lugar' o 'descabellado' plantear algo lógico: la ley como producto humano puede tanto ser cambiada como abolida.

Ni en el Reino Unido ni en Canadá, en los casos comentados, los medios de comunicación han hecho suyo el discurso que sacraliza o presenta como inmutable la ley que afecta a la unidad de su país y, en esa medida, han contribuido a un desarrollo del conflicto dentro de la categoría de la baja intensidad. En otras partes, no ha sido así.

\section{CONSIDERACIONES FINALES PARA ANALISTAS DEL DISCURSO Y DEL ROL DE LOS MEDIOS}

Por lo tanto, analizar críticamente el rol de los medios comporta asumir, en la medida de lo posible, una perspectiva con respecto al conflicto. Implica, ya sea de forma explícita o implícita, tomar una posición sobre las demandas - sobre si son o no justas o en qué medida lo son-, o sobre si los agravios son ciertos, sobre si se está favor de que haya algún tipo de conciencia colectiva -eventualmente de conciencia nacionalista-, o sobre si la dinámica con que se debe gestionar el conflicto debe ser o no violenta.

Las disyuntivas son ideológicas y, en consecuencia, admiten respuestas discutibles, no obstante, por muy ideológicas que sean, a menudo - cierto que no siempre - acaban tomando la forma de 'a favor o en contra del abuso de poder' y 'a favor 0 en contra de la escalada violenta del conflicto'. Con lo cual, no es tan difícil posicionarse.

Una vez tomada una postura [tomarla por medio de un análisis podría ser formulado como el objetivo primero/previo/cero de una investigación], es decir, después de plantear el estudio como una investigación situada -de forma similar a como se plantea en los estudios feministas-, y al hilo de las disyuntivas a que se enfrentan los periodistas, tal como hemos expuesto en este artículo, se deriva una lista de objetivos de investigación que orientativamente se podría formular, conforme Cuadro 1: 
Cuadro 1: Lista orientativa de objetivos de investigación

\begin{tabular}{|l|}
\hline Objetivo General \\
\hline \\
Identificar y discutir el análisis del conflicto \\
que ofrecen los medios (o un solo medio) \\
\hline \multicolumn{1}{|c|}{ Objos específicos } \\
\hline 1) Identificar y discutir las demandas y los agravios que el /los medios presentan \\
y qué valoraciones ofrecen; \\
2) Identificar qué identidades colectivas contribuyen a construir y con qué \\
elementos las construyen; \\
3) Analizar (y discutir) los elementos constituyentes aportados o legitimados \\
por los medios en la construcción de las identidades colectivas; \\
4) Identificar y discutir las descripciones de los actores y las responsabilidades \\
de sus acciones; \\
5) Analizar qué estrategia de resolución del conflicto promueve el medio, \\
6) [como herramienta de resumen de conjunto]: Recopilar y ordenar por \\
categorías relevantes para el conflicto, el conjunto de ideas o creencias \\
legitimadas por cada medio, o sea, reconstruir la matriz ideológica del medio \\
con relación al conflicto.
\end{tabular}

Fuente: Elaborado por el autor.

Las eventuales hipótesis solo se pueden formular después de una primera exploración del material a analizar. La exploración puede sugerir que la hipótesis sean o las siguientes (según el Cuadro 2), o sus contrarias, u otras a medio camino:

Cuadro 2: Hipótesis a formular tras la primera exploración del material de análisis

\begin{tabular}{|c|}
\hline Hipótesis General \\
\hline $\begin{array}{c}\text { El medio ofrece una visión distorsionada del } \\
\text { conflicto y de sus posibles soluciones }\end{array}$ \\
\hline Hipótesis específicas: \\
\hline 1) el discurso del medio distorsiona o falsea las demandas y los agravios \\
2) el discurso del medio construye identidades colectivas basadas en la lengua, \\
los intereses económicos, la etnia, etc. ; [escoja lo que corresponda] \\
3) el enfoque de las bases/elementos sobre las que se construyen las \\
identidades las impregna de racismo/xenofobia/odio, etc.; \\
4) unos actores son glorificados y otros son demonizados, a través de los \\
términos en que son descritos y a través de la evaluación de sus acciones o \\
presuntas acciones; \\
5) El discurso del medio promueve la escalada violenta. \\
6) la matriz ideológica muestra un medio que no construye paz ni contribuye a \\
la resolución del conflicto de forma justa y con el menos sufrimiento posible. \\
\hline
\end{tabular}

Fuente: Elaborado por el autor. 


\section{REFERENCIAS}

DAVID Trimble: Scottish nationalists "doing violence" to identity of Scots. The Guardian, London, 23 mar. 2012.

Artículo recibido el 22.03.2018 y aprobado el 02.05.2018. 